(C) 1997 International Press

Adv. Theor. Math. Phys. 1 (1997) 167-183

\title{
Partition Functions for BPS States of the Non-Critical $E_{8}$ String
}

\author{
J.A. Minahan ${ }^{a}$, D. Nemeschansky ${ }^{a}$, N.P. Warner ${ }^{b, 1}$ \\ ${ }^{a}$ Physics Department, U.S.C \\ University Park, Los Angeles, CA 90089, USA \\ ${ }^{b}$ Institute for Theoretical Physics \\ University of California \\ Santa Barbara, CA 93106-4030, USA
}

\begin{abstract}
We consider the BPS states of the $E_{8}$ non-critical string wound around one of the circles of a toroidal compactification to four dimensions. These states are indexed by their momenta and winding numbers. We find explicit expressions, $G_{n}$, for the momentum partition functions for the states with winding number $n$. The $G_{n}$ are given in terms of modular forms. We give a simple algorithm for generating the $G_{n}$, and we show that they satisfy a recurrence relation that is reminiscent of the holomorphic anomaly equations of Kodaira-Spencer theory.
\end{abstract}

\section{Introduction}

One of the surprises in the recent past is the existence of six-dimensional, $E_{8}$ non-critical strings in heterotic string theory [1]. These occur when a heterotic string is compactified on a $K 3$ manifold with a small $E_{8}$ instanton. As the instanton shrinks to zero size, the tension of a non-critical string

\footnotetext{
${ }^{1}$ On leave from Physics Department, U.S.C., University Park, Los Angeles, CA 90089.
} 
vanishes. One of the intriguing features of such strings is that they are consistent string theories that are apparently decoupled from gravity.

If one compactifies the six-dimensional theory on a circle, then one finds BPS states that correspond to momentum and winding states of the noncritical string around the compactified dimension [2,3]. After a second compactification on another circle, we are left with an $N=2$ supersymmetric theory in four dimensions, where the pre-potential has an instanton expansion whose coefficients basically count the net number of BPS states of the five-dimensional theory.

The six-dimensional heterotic theory is dual to an $F$-theory compactification on a Calabi-Yau manifold that admits an elliptic fibration. The tensionless string limit is reached when a del Pezzo 4-cycle shrinks to zero size $[4,5]$. In $[6]$ it was shown how to describe the pre-potential of the noncritical string as a function of the string tension in terms of a Seiberg-Witten theory. In [7] this was extended to incorporate other physical moduli.

In this paper, we investigate this instanton expansion as a function of two moduli: $t_{S}$ and $t_{E}$. The former is a product of the string tension and a compactification radius, and so indexes the winding states around a circle. The parameter $t_{E}$ is a background geometric factor that indexes the momentum states on the same circle. As an expansion in $t_{S}$, we find that each term of the pre-potential is an almost modular function in $t_{E}$. We explicitly compute the first few functions in the expansion. We then show that these functions satisfy a "modular anomaly" recurrence relation which is similar to the non-holomorphic recurrence relation of Kodaira-Spencer theory [8]. Using the recurrence relation we show how to generate all functions in the instanton expansion. We also have enough information about the structure of these functions to compute the BPS degeneracies in the asymptotic limit.

In section 2 we review some relevant facts from [3,6,9] and [7]. In section 3 we discuss the instanton expansion and its relation to the counting of BPS states. In section 4 we discuss and then prove the recurrence relation. We also show that the recurrence relation is very useful in computing the entire instanton expansion. In section 5 we compute the asymptotic BPS state degeneracy.

\section{The Quantum Effective Action}

\subsection{The IIA Description of the Non-Critical $E_{n}$ String}

We first briefly review some of the key elements of $[3,6]$. One considers a compactification of the IIA theory on an elliptically fibered Calabi-Yau 3-fold in which a 4-cycle is collapsing. The magnetic non-critical string may be thought of as coming from a five-brane wrapping this cycle, and the electric 
excitations of the string come from membranes wrapping the 2-cycles within the 4-cycle. One obtains the $E_{n}$ non-critical string if the collapsing 4-cycle is a del Pezzo surface, $B_{n}$, obtained by blowing-up $n$ points in $\mathbb{C P}_{2}[4,5]$.

In [3] the foregoing is realized explicitly by using a Calabi-Yau 3-fold, $\mathbf{X}_{\mathbf{F}_{\mathbf{1}}}$, that is an elliptic fibration over the Hirzebruch surface $\mathbf{F}_{\mathbf{1}}$. Since $\mathbf{X}_{\mathbf{F}_{\mathbf{1}}}$ is also a $K 3$ fibration, this compactification of the IIA theory is dual to the heterotic string compactified on $K 3 \times T_{2}$. Specifically, the corresponding heterotic string compactification has an $E_{8} \times E_{8}$ instanton embedding with $n_{1}=11, n_{2}=13$. The manifold $\mathbf{X}_{\mathbf{F}_{1}}$ has three Kähler moduli, $t_{B}, t_{E}$ and $t_{F}$, corresponding to the volumes of the base of $\mathbf{F}_{\mathbf{1}}$, the elliptic fiber of $\mathbf{X}_{\mathbf{F}_{1}}$, and the fiber of $\mathbf{F}_{\mathbf{1}}$ respectively. The modulus, $t_{B}$, is also the scale of the canonical divisor of the del Pezzo surface, and the elliptic fiber of the del Pezzo is that of $\mathbf{X}_{\mathbf{F}_{1}}$. At $t_{B}=0$ only a 2-cycle collapses, but for the whole del Pezzo to vanish one must pass through a flop transition $\left(\operatorname{Im}\left(t_{B}\right)<0\right)$ to a point where $t_{B}+t_{E}=0$.

Since $t_{B}$ is the scale of the base of the $K 3$ fibration, it follows that it must be related to the heterotic dilaton. The other two moduli, $t_{E}$ and $t_{F}$, are related to the moduli, $T=B+i R_{5} R_{6}$ and $U=e^{i \alpha} R_{5} / R_{6}$ of the torus in the heterotic compactification. In $[6,9]$ it was shown that $S, T$ and $U$ are related to the complexified Kähler moduli according to $t_{E}=U, t_{F}=T-U$ and $t_{B}=S+a T+b U$ for some undetermined constants $a, b$. The point where $t_{B}$ vanishes corresponds to an $S U(2)$ gauge symmetry enhancement at strong coupling in the heterotic string.

It is convenient to introduce a reparametrization of the moduli, replacing $t_{B}$ and $t_{F}$ by $t_{S} \equiv t_{B}+t_{E}$ and $t_{F}^{\prime}=t_{F}+t_{B}$. (Note that both these new moduli are linear in the heterotic dilaton.) From the point of view of the non-critical string, the interesting moduli are $t_{E}$ and $t_{S}$. Of the three moduli, only $t_{F}^{\prime}$ involves the tension of the fundamental heterotic string [6], and so to decouple the BPS states of fundamental heterotic string, we will consider the limit in which $t_{F}^{\prime} \rightarrow \infty$. The parameter $t_{S}$ determines the scale of the 4-cycle and hence the tension of the non-critical string. In terms of the electric BPS excitations of the non-critical string (membranes wrapping rational curves), the degrees $d_{D}$ and $d_{E}$ of the rational curve represent the winding number and momentum respectively of the non-critical string around one of the circles of the torus [3].

\subsection{Consistent Truncations}

It is fairly evident that all the physics of the non-critical string should be captured by the structure of the vanishing del Pezzo surface, and not so much by the details of the geometry of the 3-fold in which the del Pezzo surface lives. In the nearly tensionless limit, one should be able to decouple the non-critical string from ancilliary degrees of freedom like gravity. This 
decoupling should be accomplished by finding a way to abstract the del Pezzo surface from the space in which it is embedded. It was one of the key ideas in [6] that one can see how to do this in a consistent manner by passing to a closed sub-monodromy problem. That is, one identifies a basis of cycles that close (decouple from all the others) under monodromies on a special subset of moduli. Two such closed sub-monodromy problems were identified in [6], and both of them were on the other side of the flop transition $\left(\operatorname{Im}\left(t_{B}\right)<0\right)$. One finds that in the basis where the scalar fields in the vector multiplets are given by the parameters introduced above, $t_{E}, t_{S}=t_{E}+t_{B}$ and $t_{F}^{\prime}=t_{F}+t_{B}$, three of the periods depend classically upon $t_{S}$ alone, and one further period is $t_{E}$ itself.

It was further proposed in [6] that such closed sub-monodromy problems could be modeled by considering a compactification of the IIB string on a non-compact Calabi-Yau 3-fold based upon the vanishing del Pezzo surface. This non-compact Calabi-Yau manifold would thus characterize the noncritical string decoupled from the rest of the original string theory. This proposal closely parallels the way in which the quantum effective action of gauge theories (decoupled from the rest of string theory) can be obtained from ALE fibrations [10]. This idea was implemented and tested in [6], and further tested in [7], for the three period sub-monodromy problem that involves $t_{S}$ alone. Our purpose here is to generalize this to include $t_{E}$.

In a Calabi-Yau compactification of the IIB theory, the electric and magnetic BPS states are on the same footing - they come from wrapping 3-branes around $A$ or $B$-cycles of the Calabi-Yau manifold. As in [6,10], one can recast this wrapping of 3-branes in terms of wrapping a non-critical string around a Riemann surface. This is done by decomposing the 3-cycles into 2-cycles fibered over some curve in a base. The holomorhpic 3-form can then be integrated to yield some form of Seiberg-Witten differential. Thus the original pre-potential can be obtained from integrals of a meromorphic differential on a Riemann surface. For the non-critical string, such a SeibergWitten effective action encodes all the counting of rational curves in the del Pezzo surface $[6,7]$. In $[6,7]$ this was done for sub-monodromy problem involving $t_{S}$ alone, which yields information about the BPS states with $d_{D}=d_{E}$. These states were focussed on because they were, in a sense, the most stringy: they are the states that become massless when the non-critical string becomes tensionless. The reduction of the problem from a 3-fold to a torus was also critical to [7] in that it enabled the simple generalization to include Wilson lines, and led to a conjecture as to how to include $t_{E}$.

The starting point of $[6,7]$ was to consider the IIB string theory compactified on the non-compact Calabi-Yau 3-fold defined by

$$
w^{2}=z_{1}^{3}+z_{2}^{6}+z_{3}^{6}-\frac{1}{z_{4}^{6}}-\psi w z_{1} z_{2} z_{3} z_{4} .
$$


The modulus $\psi$ determines the string tension. As described in [6], the holomorphic 3-form on this manifold has three periods corresponding to terms in the BPS mass formula. With suitable normalization, the period integrals can be identified with $1, t_{S}$ and $\partial \mathcal{F} / \partial t_{S}$. This gives the truncation of the non-critical string to the sector in which $t_{S}$ is the only parameter, and in which the BPS states have $d_{E}=d_{D}$.

To get the states of the string with independent winding number and momentum $\left(d_{D}\right.$ and $\left.d_{E}\right)$ we want the slightly less stringent truncation described in [6]: one in which the period integrals are $1, t_{S}, t_{E}$ and $\partial \mathcal{F} / \partial t_{S}$. The relevant manifold was proposed in [7]:

$$
w^{2}=z_{1}^{3}+z_{2}^{6}+z_{3}^{6}-\frac{1}{z_{4}^{6}}+\psi^{2}\left(1+k^{2}\right)\left(z_{1} z_{2} z_{3} z_{4}\right)^{2}+k^{2} \psi^{4} z_{1}\left(z_{2} z_{3} z_{4}\right)^{4} .
$$

The moduli are $\psi$ and $k$, and 2.2 reduces to 2.1 in the limit $k \rightarrow 0$ (one also has to shift $w$ and re-scale $\psi$ ). It is convenient to think of $k$ as the modulus of a set of Jacobi elliptic functions, and so we parametrize $k$ in terms of another variable, $\tau$, via

$$
k \equiv \vartheta_{2}^{2}(0 \mid \tau) / \vartheta_{3}^{2}(0 \mid \tau) .
$$

The holomorphic 3-form can be represented as

$$
\Omega=\frac{\psi}{2 \omega_{2}} \frac{z_{4} d z_{1} d z_{2} d z_{3}}{w}
$$

where the (constant) normalization factor $\frac{\psi}{2 \omega_{2}}$ will be defined below. To isolate the relevant periods one now follows the approach of [6]: Go to a patch with $z_{4}=1$, and make a change of variables $z_{1}=\zeta z_{2}^{2}$. Considered as a function of $z_{2}, \Omega$ has branch cuts. These cuts disappear when $z_{3}^{6}=1$. Integrate $z_{2}$ around a circle around all these cuts. Let $\xi=\psi z_{3}$, and then the period integrals reduce to:

$$
\frac{1}{2 \omega_{2}} \int \frac{d \xi d \zeta}{\sqrt{\zeta^{3}+1-\left(1+k^{2}\right) \zeta^{2} \xi^{2}+k^{2} \zeta \xi^{4}}} .
$$

There are two types of period integral:

1. (i) Integrate $\xi$ between roots of $\xi^{6}=\psi^{6}$

2. (ii) Take $\zeta=x \xi^{2}$ and integrate $\xi$ around a circle of large radius.

Doing the latter first, one is left with a standard elliptic integral in $x$ :

$$
\frac{1}{\omega_{2}} \int \frac{d x}{\sqrt{\left(x^{3}-\left(1+k^{2}\right) x^{2}+k^{2} x\right)}}=\frac{1}{\omega_{2}} \int \frac{d t}{\sqrt{\left(1-t^{2}\right)\left(1-k^{2} t^{2}\right)}},
$$


where $x=1 / t^{2}$.

Shifting $x$, one can recast the elliptic integral in standard Weierstrass form $\int d x / y$ with $y^{2}=4 x^{3}-g_{2} x-g_{3}$ and

$$
g_{2}=\frac{4}{3}\left(1-k^{2}+k^{4}\right), \quad g_{3}=\frac{4}{27}\left(1+k^{2}\right)\left(2 k^{2}-1\right)\left(k^{2}-2\right) .
$$

The periods of the torus defined by $x$ and $y$ are denoted by $\omega_{1}$ and $\omega_{2}$. This defines $\omega_{2}$ in 2.4, and with this normalization the periods 2.6 are 1 and $\tau=\omega_{1} / \omega_{2}$. The parametrization 2.3 gives the relationship between $k$ and the parameter $\tau$ introduced here. The parameter $\tau$ is to be identified with the Kähler modulus $t_{E}$.

Returning to the other period integrals, we first recast them into a form similar to that of [7]. Make a change of variables $\zeta=\frac{1}{4} x / \xi^{10}, u=-2 \xi^{6}$. The integral becomes $\frac{i}{12 \omega_{2}} \int d x d u / y$ where

$$
y^{2}=x^{3}+\left(1+k^{2}\right) u^{2} x^{2}+k^{2} u^{4} x-2 u^{5} .
$$

This can easily be recast into standard Weierstrass form $y^{2}=4 x^{3}-\tilde{g}_{2} x-\tilde{g}_{3}$ with:

$$
\begin{aligned}
& \tilde{g}_{2}=\frac{4}{3}\left(1-k^{2}+k^{4}\right) u^{4}=g_{2} u^{4}, \\
& \tilde{g}_{3}=\frac{4}{27}\left(1+k^{2}\right)\left(2 k^{2}-1\right)\left(k^{2}-2\right) u^{6}-8 u^{5}=g_{3}-8 u^{5} .
\end{aligned}
$$

Let $\tilde{\omega}_{i}$ be the periods of this torus, and $\tilde{\tau}=\tilde{\omega}_{1} / \tilde{\omega}_{2}$. The periods we then seek are the indefinite integrals $\int d u \tilde{\omega}_{i} / \omega_{2}$ where $u=-2 \psi^{6}$.

Introduce the Eisenstein functions:

$$
\begin{aligned}
& E_{2}(\tau) \equiv 1-24 \sum_{n=1}^{\infty} \sigma_{1}(n) q^{n}, \\
& E_{4}(\tau) \equiv 1+240 \sum_{n=1}^{\infty} \sigma_{3}(n) q^{n}, \\
& E_{6}(\tau) \equiv 1-504 \sum_{n=1}^{\infty} \sigma_{5}(n) q^{n},
\end{aligned}
$$

where $q=e^{2 \pi i \tau}$ and $\sigma_{p}(n)$ is the sum of the $p^{\text {th }}$ powers of the divisors of $n$. One should recall that $E_{4}$ and $E_{6}$ transform as modular functions of weight 4 and 6 respectively, and that

$$
E_{2}\left(\frac{a \tau+b}{c \tau+d}\right)=(c \tau+d)^{2}\left(E_{2}(\tau)+\frac{6}{\pi i} \frac{c}{c \tau+d}\right) .
$$

From 2.9 and the standard relationship between the periods of a torus and the Eisenstein functions one finds:

$$
\tilde{\omega}_{2}=\frac{\omega_{2}}{u}\left(\frac{E_{4}(\tilde{\tau})}{E_{4}(\tau)}\right)^{1 / 4}, \quad \frac{E_{4}^{3}(\tilde{\tau})}{E_{6}^{2}(\tilde{\tau})}=\frac{E_{4}^{3}(\tau)}{\left(E_{6}(\tau)-v\right)^{2}},
$$

where $v=\frac{1}{27}\left(\frac{\pi}{\omega_{2}}\right)^{6} \frac{1}{u}$. The second equation in 2.12 may be used to determine $\tilde{\tau}$ as a function of $\tau$ and $u$. This can then be substituted into the first 
equation to give $\tilde{\omega}_{2} / \omega_{2}$ in terms of $\tau$ and $u$. One then gets the other period integral from $\tilde{\omega}_{1} / \omega_{2}=\tilde{\tau} \tilde{\omega}_{2} / \omega_{2}$.

Therefore, the other two periods of $\Omega$ are:

$$
\varphi \equiv \frac{1}{2 \pi i} \int \frac{d v}{v}\left(\frac{E_{4}(\tilde{\tau})}{E_{4}(\tau)}\right)^{1 / 4} ; \varphi_{D} \equiv \frac{1}{2 \pi i} \int \frac{d v}{v} \tilde{\tau}\left(\frac{E_{4}(\tilde{\tau})}{E_{4}(\tau)}\right)^{1 / 4}
$$

where $\tau$ is fixed, and $\tilde{\tau}$ is obtained from 2.12 .

\section{Curve Counting and the Instanton Expansion}

To count the BPS states we want the Taylor expansion of the periods 2.13 about $v=0$. To evolve these series it is useful to recall that:

$$
\begin{aligned}
E_{2} & =q \frac{d}{d q} \log (\Delta) ; \quad q \frac{d}{d q} E_{2}=\frac{1}{12}\left(E_{2}^{2}-E_{4}\right) ; \\
q \frac{d}{d q} E_{4} & =\frac{1}{3}\left(E_{2} E_{4}-E_{6}\right) ; \quad q \frac{d}{d q} E_{6}=\frac{1}{2}\left(E_{2} E_{6}-E_{4}^{2}\right)
\end{aligned}
$$

where $\Delta=\eta^{24}=q \prod_{n=1}^{\infty}\left(1-q^{n}\right)^{24}=\frac{1}{1728}\left(E_{4}^{3}-E_{6}^{2}\right)$. Also recall that the modular invariant $j$ is given by $j=E_{4}^{3} / \Delta$. From 2.12 one obtains:

$$
\tilde{\tau}=\tau+\sum_{j=1}^{\infty} a_{j} v^{j}
$$

and the $a_{j}$ can be obtained using 3.1. The first few terms are:

$$
\begin{aligned}
& a_{1}=2 E_{4} / \Delta, \quad a_{2}=-\frac{1}{3 \Delta^{2}} E_{4}\left(5 E_{6}+E_{2} E_{4}\right), \\
& a_{3}=-\frac{1}{\Delta^{3}} E_{4}\left(\frac{31}{54} E_{4}^{3}+\frac{40}{27} E_{6}^{2}+\frac{5}{9} E_{2} E_{4} E_{6}+\frac{1}{18} E_{2}^{2} E_{4}^{2}\right) .
\end{aligned}
$$

Note that the second equation in 2.12 is invariant under the combined modular transformations:

$$
\tau \rightarrow \frac{a \tau+b}{c \tau+d}, \quad \tilde{\tau} \rightarrow \frac{a \tilde{\tau}+b}{c \tilde{\tau}+d}, \quad v \rightarrow(c \tau+d)^{6} v .
$$

The fact that one must simultaneously transform $\tau$ and $\tilde{\tau}$ follows from requiring an expansion of the form 3.2. It is also easy to verify that the function

$$
\varphi_{D}-\tau \varphi=\frac{1}{2 \pi i} \int \frac{d v}{v}(\tilde{\tau}-\tau)\left(\frac{E_{4}(\tilde{\tau})}{E_{4}(\tau)}\right)^{1 / 4} \equiv \sum_{j=1}^{\infty} b_{j} v^{j}
$$

considered as a function of $\tau$ and $v$, transforms as a modular function of weight -2 under 3.4. Thus upon substituting 3.2 into 3.5 , and expanding in a power series is $v$, the coefficient, $b_{j}$, of $v^{j}$ must be an exactly modular 
function of weight $-2-6 j$. One also sees from 2.12 that the only pole in $\varphi_{D}-\tau \varphi$ is at $\tau=i \infty$, and from examining the form of $a_{j}$, one sees that the $b_{j}$ must be of the form $P_{6 j-2}\left(E_{4}, E_{6}\right) / \Delta^{j}$, where $P_{6 j-2}\left(E_{4}, E_{6}\right)$ is a polynomial in $E_{4}$ and $E_{6}$ alone, such that it has modular weight $6 j-2$.

Up to "constants" of integration, $\delta(\tau)$ and $\delta_{D}(\tau)$, (which could be arbitrary functions of $\tau$ ), the periods $\varphi$ and $\varphi_{D}$ are $t_{S}$ and $\partial \mathcal{F} / \partial t_{S}$ :

$$
t_{S}=\varphi-\delta(\tau) ; \quad \frac{\partial \mathcal{F}}{\partial t_{S}}=\varphi_{D}-\delta_{D}(\tau) .
$$

Let $q_{S}=e^{2 \pi i t_{S}}$ and recall $q=e^{2 \pi i \tau}=e^{2 \pi i t_{E}}$. Define $C_{t_{S} t_{S} t_{S}}=\frac{\partial^{3} \mathcal{F}}{\partial t_{S}^{3}}$, and recall that it has an instanton expansion:

$$
C_{t_{S} t_{S} t_{S}}=\sum_{n_{1}, n_{2}}(-1)^{n_{1}+1} N_{n_{1}, n_{2}} \frac{n_{1}^{3} q_{S}^{n_{1}} q^{n_{2}}}{1-q_{S}^{n_{1}} q^{n_{2}}}
$$

where $N_{n_{1}, n_{2}}$ is the number of rational curves with $d_{D}=n_{1}$ and $d_{E}=n_{1}+n_{2}$.

To develop the instanton expansion we therefore have to substitute 3.2 into the expression for $\varphi$, and expand the series in $v$. One then inverts this series to obtain a series for $v$ in powers of $e^{2 \pi i \varphi}$, with coefficients that are functions of $\tau$. This is then substituted into the series expansion for $C_{t_{S} t_{S} t_{S}}$. The result is a series of the form:

$$
C_{t_{S} t_{S} t_{S}}=\sum_{n=1}^{\infty} \widetilde{F}_{n}(\tau) e^{2 \pi i n \varphi}=\sum_{n=1}^{\infty} F_{n}(\tau) q_{S}^{n}
$$

where $F_{n}(\tau)=\widetilde{F}_{n}(\tau) e^{2 \pi i n \delta(\tau)}$. It is trivial to see that $\widetilde{F}_{1}(\tau)=a_{1}(\tau)$, where $a_{1}$ is given in 3.3. The first term in this series 3.8 was computed in [3], and is given by:

$$
F_{1}(\tau)=\frac{E_{4}}{q^{1 / 2} \eta^{12}} .
$$

(The factor in the denominator is $q^{1 / 2}$ and not $q^{-1 / 2}$, since this is the coefficient of $q_{S}=q e^{2 \pi i t_{D}}$.) This fixes $\delta(\tau)=\frac{1}{2 \pi i} \log \left(-\frac{1}{2} q^{-1 / 2} \eta^{12}\right)$.

Using Mathematica ${ }^{T M}$, one can easily compute the $F_{n}$ to fairly high order ( $n=12$ ). Defining $G_{n}=q^{n / 2} F_{n}$, one finds that $G_{n}$ has the form of

$$
G_{n}=Q_{6 n-2}\left(E_{2}, E_{4}, E_{6}\right) / \Delta^{n / 2}
$$

where $Q_{6 n-2}$ is a polynomial of degree $6 n-2$ (where $E_{2}, E_{4}$ and $E_{6}$ are given weights 2,4 and 6 respectively). Because of the presence of $E_{2}$ and the half powers of $\Delta$, the functions $G_{n}$ are not quite modular functions of weight -2 . 
The first few $G_{n}$ are:

$$
\begin{gathered}
G_{1}=\frac{E_{4}}{\Delta^{1 / 2}}, \quad G_{2}=\frac{E_{4}\left(E_{2} E_{4}+2 E_{6}\right)}{3 \Delta}, \\
G_{3}=\frac{1}{576 \Delta^{\frac{3}{2}}} E_{4}\left(54 E_{2}{ }^{2} E_{4}{ }^{2}+109 E_{4}{ }^{3}+216 E_{2} E_{4} E_{6}+197 E_{6}{ }^{2}\right) \\
G_{4}=\frac{1}{972 \Delta^{2}} E_{4}\left(\begin{array}{c}
24 E_{2}^{3} E_{4}{ }^{3}+109 E_{2} E_{4}{ }^{4}+144 E_{2}^{2} E_{4}^{2} E_{6}+ \\
\left.272 E_{4}{ }^{3} E_{6}+269 E_{2} E_{4} E_{6}{ }^{2}+154 E_{6}{ }^{3}\right)
\end{array}\right. \\
G_{5}=\frac{1}{2985984 \Delta^{\frac{5}{2}} E_{4}}\left(18750 E_{2}{ }^{4} E_{4}{ }^{4}+136250 E_{2}{ }^{2} E_{4}{ }^{5}+116769 E_{4}{ }^{6}+\right. \\
150000 E_{2}{ }^{3} E_{4}{ }^{3} E_{6}+653000 E_{2} E_{4}{ }^{4} E_{6}+ \\
426250 E_{2}{ }^{2} E_{4}{ }^{2} E_{6}{ }^{2}+772460 E_{4}{ }^{3} E_{6}{ }^{2}+ \\
\left.505000 E_{2} E_{4} E_{6}{ }^{3}+207505 E_{6}{ }^{4}\right) \\
G_{6}=\frac{1}{74649600 \Delta^{3} E_{4}}\left(116640 E_{2}^{5} E_{4}^{5}+1177200 E_{2}^{3} E_{4}^{6}+2398867 E_{2} E_{4}{ }^{7}+\right. \\
1166400 E_{2}^{4} E_{4}^{4} E_{6}+8229600 E_{2}^{2} E_{4}^{5} E_{6}+ \\
6703718 E_{4}{ }^{6} E_{6}+{ }^{4} 460400 E_{2}{ }^{3} E_{4}{ }^{3} E_{6}{ }^{2}+ \\
18894730 E_{2} E_{4}{ }^{4} E_{6}{ }^{2}+8100000 E_{2}{ }^{2} E_{4}{ }^{2} E_{6}^{3}+ \\
14280020 E_{4}{ }^{3} E_{6}{ }^{3}+6922915 E_{2} E_{4} E_{6}{ }^{4}+ \\
\left.2199110 E_{6}{ }^{5}\right) \cdot
\end{gathered}
$$

These functions are completely consistent with the numbers generated in [3].

The first function in 3.11 has the simple interpretation as the $E_{8}$ root lattice partition function multiplied by the partition function of four bosonic oscillators coming from the space-time [3]. The second function is almost as simple: it can be rewritten as $G_{1} G_{1}^{\prime}$, where $G_{1}^{\prime}=q \frac{q}{d q} G_{1}$. Since this partition function represents momentum excitations of a doubly wound string, one naively expects a tensor product, or $G_{1}^{2}$. However, since it is a bound state, it cannot be a simple tensor product. The derivative with respect to $\tau$ pulls down a Hamiltonian and thus removes one of the two independent translation invariances of the doubly wound state. Unfortunately the higher $G_{n}$ do not appear to admit such a simple interpretation. However, as we will see in the next section, the $G_{n}$ are indeed related to one another.

\section{Modular Properties and a Recurrence Relation}

There is no a priori reason to expect the non-critical string to exhibit $T$-duality, but the $G_{n}$ are almost modular functions (of weight -2 ), and so the spectrum of BPS states is almost invariant under $\tau \rightarrow \frac{a \tau+b}{c \tau+d}$. There are three places in which the BPS spectrum fails to be modular invariant: 
(i) the bare factors of $q$ in $F_{n}=q^{n / 2} G_{n}$, (ii) the odd powers of $\Delta^{1 / 2}$ in $G_{n}$, and (iii) the anomalous modular behaviour of $E_{2}$. The first problem has a trivial cure: one simply redefines $t_{S}$. The second problem means that one really has only a subgroup of the modular groups as a symmetry. Alternatively, one can work with the full modular group, and remember to make appropriate changes of sign in the odd powers of $\Delta^{1 / 2}$. The anomalous behaviour of $E_{2}$ also has a cure, but at the cost of holomorphy. That is, the function

$$
\widehat{E_{2}}(\tau)=E_{2}(\tau)-\frac{3}{\pi} \frac{1}{\operatorname{Im}(\tau)}
$$

satisfies

$$
\widehat{E_{2}}\left(\frac{a \tau+b}{c \tau+d}\right)=(c \tau+d)^{2} \widehat{E_{2}}(\tau) .
$$

Define $\widehat{\mathcal{G}}_{n}$ to be $G_{n} / n^{3}$ but with $E_{2}$ replaced by $\widehat{E_{2}}$, and introduce:

$$
\widehat{\mathcal{G}}(\sigma, \tau)=\sum_{n=1}^{\infty} \widehat{\mathcal{G}}_{n}(\tau) e^{2 \pi i n \sigma}
$$

One can recover the instanton expansion from this by taking three derivatives with respect to $\sigma$, setting $\sigma=t_{S}+\frac{1}{2} \tau$, and by sending $\bar{\tau} \rightarrow \infty$ while holding $\tau$ fixed. The function $\widehat{\mathcal{G}}$ is also modular invariant. To this extent the noncritical string exhibits a $T$-duality.

The function $\widehat{\mathcal{G}}$ exhibits another remarkable property: it satisfies a recurrence relation that is reminiscent of the holomorphic anomaly equation in Kodaira-Spencer theory [8]. This follows from a "modular anomaly" recurrence relation that is satisfied by the $F_{n}$. Let $f_{n}=F_{n} / n^{3}$, and view it as a function of the variables $E_{2}, E_{4}$ and $E_{6}$; then we will show that

$$
\frac{\partial f_{n}}{\partial E_{2}}=\frac{1}{24} \sum_{m=1}^{n-1} m(n-m) f_{m} f_{n-m} .
$$

If one replaces $E_{2}$ by $\widehat{E_{2}}$, one has $\frac{\partial}{\partial \bar{\tau}}=-\frac{3 i}{2 \pi} \frac{1}{(\operatorname{Im}(\tau))^{2}} \frac{\partial}{\partial \widehat{E}_{2}}$. Hence it follows that $\widehat{\mathcal{G}}$ satisfies:

$$
\frac{\partial \widehat{\mathcal{G}}}{\partial \bar{\tau}}=-\frac{i}{16 \pi} \frac{1}{(\operatorname{Im}(\tau))^{2}} \frac{\partial \widehat{\mathcal{G}}}{\partial \sigma} \frac{\partial \widehat{\mathcal{G}}}{\partial \sigma}
$$

This equation is almost exactly of the form of the holomorphic anomaly equation of [8], although the physical origins of the problem are rather different. In [8] the holomorphic anomaly was used to relate partition functions on higher genus Riemann surfaces to those of lower genus. In our case, the anomaly equations are used to relate multi-instanton expansions to lower 
instanton terms. This probably explains one key difference between the anomaly equations. In [8], the anomaly equation for $F_{g}$, where $g$ is the genus, contains the piece $\partial^{2} F_{g-1}$. Such a term arises from pinching off a handle on the Riemann surface. It is hard to imagine an analogous process for a multi-instanton.

Still, the similarities are striking. The instanton expansion consists of maps onto a target space that is a torus with modulus $\tau$. The factor of $1 /(\operatorname{Im}(\tau))^{2}$ in 4.5 has the interpretation of a metric $g^{\sigma \sigma}$ on the torus [8], while the fact that the differential equation involves a derivative with respect to $\bar{\tau}$ on the left and derivatives with respect to $\sigma$ on the right is consistent with the form of the classical intersection form: Since $\varphi_{D}=\tau \varphi$ it follows that $C_{\tau t_{S} t_{S}}=1$. There is no corresponding equation for $\partial_{\bar{\phi}} \widehat{\mathcal{G}}$ since the classical (non-instanton) part of $C_{t_{S} t_{S} t_{S}}$ is zero. Given this close correspondence, it is tempting to conjecture that $e^{2 \pi i t_{S}}$ might be a loop expansion parameter for the non-critical string.

The recurrence relation in 4.4 turns out to be a powerful tool in computing the $G_{n}$ of the previous section. Given the lower $G_{m}$, the recurrence relation determines $G_{n}(n>m)$ up to a piece $E_{4} K_{n} / \Delta^{n / 2}$, where $K_{n}$ is a modular form of weight $6(n-1)$. The space of such forms has dimension $[(n+1) / 2]$, thus to completely determine $G_{n}$, we need to compute $[(n+1) / 2]$ coefficients. We actually have more than enough information to do this, since we know that the $q$ expansion of $G_{n}$ has the form ${ }^{2} G_{n}=q^{-n / 2}+\mathrm{O}\left(q^{n / 2}\right)$. Hence, all we need to do is adjust the coefficients in $K_{n}$, such that the leading term in $G_{n}$ is $q^{-n / 2}$ and the rest of the negative powers in $G_{n}$ have coefficients that are zero. A useful check is that the nonnegative powers up to the $q^{n / 2}$ term also have zeros for coefficients. Using Mathematica ${ }^{T M}$, we can easily generate the $K_{n}$, the first 12 of which are given by

$$
\begin{aligned}
K_{1}= & 1, \quad K_{2}=\frac{2 E_{6}}{3}, \quad K_{3}=\frac{1}{576}\left(109 E_{4}{ }^{3}+197 E_{6}{ }^{2}\right) \\
K_{4}= & \frac{E_{6}}{486}\left(136 E_{4}{ }^{3}+77 E_{6}{ }^{2}\right) \\
K_{5}= & \frac{1}{2985984}\left(116769 E_{4}{ }^{6}+772460 E_{4}{ }^{3} E_{6}{ }^{2}+207505 E_{6}{ }^{4}\right) \\
K_{6}= & \frac{E_{6}}{37324800}\left(3351859 E_{4}{ }^{6}+7140010 E_{4}{ }^{3} E_{6}{ }^{2}+1099555 E_{6}{ }^{4}\right) \\
K_{7}= & \frac{1}{386983526400}\left(3214033725 E_{4}{ }^{9}+46377519701 E_{4}{ }^{6} E_{6}{ }^{2}+\right. \\
& \left.\quad+47881472765 E_{4}{ }^{3} E_{6}{ }^{4}+4721253845 E_{6}{ }^{6}\right), \\
K_{8}= & \frac{E_{6}}{111106598400}\left(2874313704 E_{4}{ }^{9}+13496101157 E_{4}{ }^{6} E_{6}{ }^{2}+\right. \\
& \left.+8126197310 E_{4}{ }^{3} E_{6}{ }^{4}+551920565 E_{6}{ }^{6}\right)
\end{aligned}
$$

\footnotetext{
${ }^{2}$ This follows from 3.7 and the fact that $n_{1} \geq 1 n_{2} \geq 0$ and $N_{1,0}=1$.
} 


$$
\begin{aligned}
K_{9}= & \frac{1}{1213580338790400}\left(2168558256025 E_{4}{ }^{12}+54762568177568 E_{4}{ }^{9} E_{6}{ }^{2}\right. \\
& +125727877850316 E_{4}{ }^{6} E_{6}{ }^{4}+49166052530000 E_{4}{ }^{3} \\
& \left.+E_{6}{ }^{6}+2423088666145 E_{6}{ }^{8}\right) \\
K_{10}= & \frac{E_{6}}{88470006697820160}\left(621851537315031 E_{4}{ }^{12}+5138509650200980 E_{4}{ }^{9} E_{6}{ }^{2}\right. \\
& +6932453167897530 E_{4}{ }^{6} E_{6}{ }^{4}+1889989579331700 E_{4}{ }^{3} E_{6}{ }^{6} \\
& \left.+70283214345575 E_{6}{ }^{8}\right) \\
K_{11}= & \frac{1}{4246560321495367680000}\left(16449098432914744375 E_{4}{ }^{15}\right. \\
& +64293839773877897511 E_{4}{ }^{12} E_{6}{ }^{2}+261044867981347260580 E_{4}{ }^{9} E_{6}{ }^{4} \\
& +230239896247913645940 E_{4}{ }^{6} E_{6}{ }^{6}+46023695034064491975 E_{4}{ }^{3} E_{6}{ }^{8} \\
& \left.+1331341121000896775 E_{6}{ }^{10}\right), \\
K_{12}= & \frac{E_{6}}{5352435405218119680000}\left(9853164552615074200 E_{4}^{15}\right. \\
& +126458286011220239911 E_{4}{ }^{12} E_{6}{ }^{2}+303024347024677902580 E_{4}{ }^{9} E_{6}{ }^{4} \\
& +187415787390550146890 E_{4}{ }^{6} E_{6}{ }^{6}+28525664977566703100 E_{4}{ }^{3} E_{6}{ }^{8} \\
& \left.+657760456052320775 E_{6}{ }^{10}\right) .
\end{aligned}
$$

The coefficients of these polynomials are always positive. At this time, we know of no other system where these particular modular forms appear naturally.

We conclude by proving the recurrence relation 4.4. This relation is equivalent to:

$$
\frac{\partial}{\partial E_{2}}\left(\varphi_{D}-\tau \varphi\right)=\frac{2 \pi i}{24} \frac{\partial}{\partial \varphi}\left(\varphi_{D}-\tau \varphi\right)^{2},
$$

where $\varphi_{D}-\tau \varphi$ is viewed as a function of $\varphi, E_{2}, E_{4}$ and $E_{6}$. Now recall that the coefficients $b_{j}$ in 3.5 are independent of $E_{2}$, and so $\varphi_{D}-\tau \varphi$ inherits its $E_{2}$ dependence only through the implicit dependence of $v$ on $\varphi, E_{2}, E_{4}$ and $E_{6}$. As a result, 4.7 , and hence 4.4 , is equivalent to

$$
\frac{\partial v}{\partial E_{2}}=\frac{2 \pi i}{12}\left(\varphi_{D}-\tau \varphi\right) \frac{\partial v}{\partial \varphi} \quad \text { or } \quad\left(\varphi_{D}-\tau \varphi\right)=\frac{12}{2 \pi i} \frac{\partial_{E_{2}} v}{\partial_{\varphi} v}
$$

Differentiating the first equation in 2.13 with respect to both $\varphi$ and $E_{2}$ yields

$$
\begin{aligned}
2 \pi i & =\frac{\partial_{\varphi} v}{v}\left(\frac{E_{4}(\tilde{\tau})}{E_{4}(\tau)}\right)^{1 / 4}, \\
0 & =\frac{\partial_{E_{2}} v}{v}\left(\frac{E_{4}(\tilde{\tau})}{E_{4}(\tau)}\right)^{1 / 4}+\int^{v} \frac{d v}{v} \partial_{E_{2}}\left(\frac{E_{4}(\tilde{\tau})}{E_{4}(\tau)}\right)^{1 / 4}
\end{aligned}
$$


where the $E_{2}$ derivative acting on the integrand is for the explicit $E_{2}$ dependence (i.e. not the implicit dependence in $v$ ). From these two equations one obtains:

$$
\frac{\partial_{E_{2}} v}{\partial_{\varphi} v}=-\frac{1}{2 \pi i} \int^{v} \frac{d v}{v} \partial_{E_{2}}\left(\frac{E_{4}(\tilde{\tau})}{E_{4}(\tau)}\right)^{1 / 4}
$$

Using this in 4.8 one sees that the recurrence relation is equivalent to:

$$
\int^{v} \frac{d v}{v}\left(\frac{E_{4}(\tilde{\tau})}{E_{4}(\tau)}\right)^{1 / 4}(\tilde{\tau}-\tau)=-\frac{12}{2 \pi i} \int^{v} \frac{d v}{v} \partial_{E_{2}}\left(\frac{E_{4}(\tilde{\tau})}{E_{4}(\tau)}\right)^{1 / 4}
$$

or equating integrands, one has

$$
\left(\frac{E_{4}(\tilde{\tau})}{E_{4}(\tau)}\right)^{1 / 4}(\tilde{\tau}-\tau)=-\frac{12}{2 \pi i} \partial_{E_{2}}\left(\frac{E_{4}(\tilde{\tau})}{E_{4}(\tau)}\right)^{1 / 4}
$$

Since $\left(\frac{E_{4}(\tilde{\tau})}{E_{4}(\tau)}\right)^{1 / 4}(\tilde{\tau}-\tau)$ is a modular function of weight -2 , it cannot have any explicit $E_{2}$ dependence. Using this fact in the right hand side of 4.12 means that 4.12 is equivalent to:

$$
\partial_{E_{2}}(\tilde{\tau}-\tau)=-\frac{2 \pi i}{12}(\tilde{\tau}-\tau)^{2} .
$$

Thus proving 4.13 is equivalent to establishing the recurrence relation. Let $H=\tilde{\tau}-\tau$, then under the modular transformation 3.4,

$$
H=(\tilde{\tau}-\tau) \rightarrow \frac{(\tilde{\tau}-\tau)}{(c \tau+d)(c \tilde{\tau}+d)}=\frac{H /(c \tau+d)^{2}}{1+c H /(c \tau+d)} .
$$

Let $h$ be defined by

$$
H=\frac{h}{1+\frac{2 \pi i}{12} E_{2} h} .
$$

Then 4.14 and 2.11 implies that $h \rightarrow(c \tau+d)^{-2} h$, i.e. it is a modular function of weight -2 . It therefore cannot have any explicit $E_{2}$ dependence, and so from 4.15 one has:

$$
\partial_{E_{2}} H=-\frac{2 \pi i}{12} \frac{h^{2}}{\left(1+\frac{2 \pi i}{12} E_{2} h\right)^{2}}=-\frac{2 \pi i}{12} H^{2} .
$$

This establishes 4.13 , and hence proves the recurrence relation.

\section{Asymptotic Degeneracies of BPS States}

An important question is the asymptotic behaviour for the degeneracies of BPS states, $N_{n_{1}, n_{2}}$, as $n_{1}$ and $n_{2}$ approach large values in 3.7. By knowing 
the behaviour of the degeneracies one can compare the entropy with that of other interesting physical systems.

The contributions of multicoverings becomes negligible in the asymptotic limit, so to a very good approximation

$$
N_{n_{1}, n_{2}}=(-1)^{n_{1}+1} F_{n_{1}, n_{2}} / n_{1}^{3},
$$

where $F_{n_{1}, n_{2}}=G_{n_{1}, n_{2}+n_{1} / 2}$ is the coefficient of $q^{n_{2}}$ in $F_{n_{1}}$. The coefficient $G_{n, m}$ is given by

$$
G_{n, m}=\frac{1}{2 \pi i} \oint \frac{d w}{w^{m+1}} G_{n}(\tau)
$$

where $w=e^{2 \pi i \tau}$, and the integral is taken around any circle centered on the origin in the $w$-plane. We now follow the method in [11] of making a saddle point approximation of this integral for large values of $m$. To do this we first recall the normalization requirement that $G_{n} \sim 1 . q^{-n / 2}$ as $q \rightarrow 0$. To get a well-behaved and consistent approximation, one first makes a modular inversion, and considers the limit in which $\tau$ is small. Recall that $E_{4}$ and $E_{6}$ are modular forms of weight 4 and $6, E_{2}$ transforms as in 2.11 , and that $\Delta^{1 / 2}(-1 / \tau)=-\tau^{6} \Delta^{1 / 2}(\tau)$. If one keeps only the leading terms in $e^{-2 \pi i / \tau}$, one can neglect the anomalous modular pieces of the transformation of $E_{2}$, and so with the exception of the sign in the transformation of $\Delta^{1 / 2}, G_{n}$ to leading order behaves like a modular function of weight -2 . Keeping only the leading term in the $q$-expansion of $G_{n}$ then yields:

$$
G_{n, m} \approx \frac{1}{2 \pi i} \oint \frac{d w}{w^{m+1}}(-1)^{n}\left(-\frac{2 \pi i}{\log w}\right)^{2} \exp \left(\frac{-4 \pi^{2} n}{2 \log w}\right) .
$$

The saddle point is at $\tau=i \sqrt{\frac{n}{2 m}}$, which is indeed small as $m$ becomes large. Continuing in the usual way with the standard saddle point approximation, we find

$$
G_{n, m} \approx-(-1)^{n} \frac{n^{5 / 4}}{(2 m)^{7 / 4}} \exp (2 \pi \sqrt{2 n m}) .
$$

Hence, the asymptotic approximation for $N_{n_{1}, n_{2}}$ is

$$
N_{n_{1}, n_{2}} \approx\left(n_{1}^{2}+2 n_{1} n_{2}\right)^{-7 / 4} \exp \left(2 \pi \sqrt{n_{1}^{2}+2 n_{1} n_{2}}\right)
$$

which is valid for both $n_{1}$ and $n_{2}$ large.

We can also consider the limit where $n_{1}$ is large and $n_{2}$ is zero. From 5.5 , the naive behaviour is

$$
N_{n_{1}, 0} \sim\left(n_{1}\right)^{-7 / 2} \exp \left(2 \pi n_{1}\right) .
$$


Let us compare this with the exact asymptotic behaviour. We can project onto the $n_{2}=0$ states by letting $\tau \rightarrow i \infty$. This reduces the model to the case considered in [6] and [7]. Now the instanton expansion is given by

$\varphi_{D}=-\frac{1}{2} \varphi^{2}+\frac{1}{2} \varphi+\frac{5}{12}-\sum_{n=1} \frac{C_{n}}{4 \pi^{2} n^{2}} e^{2 \pi i n \varphi}, \quad \partial_{\varphi}^{3} \varphi_{D}=\sum_{n=1} 2 \pi i n C_{n} e^{2 \pi i n \varphi}$.

For the asymptotic expansion we can again ignore the multicoverings. Thus the degeneracy $d_{n}$ is

$$
d_{n} \approx-(-1)^{n} C_{n} / n^{3} .
$$

The instanton expansion diverges at the conifold point $u=1$. Near this point $\varphi$ and $\varphi_{D}$ are approximately [6]:

$$
\begin{aligned}
\varphi & =c-\frac{i}{2 \pi}\left(\phi_{D}-1\right) \log (u-1)+\ldots \\
\varphi_{D} & =1+\frac{1}{2 \pi}(u-1) .
\end{aligned}
$$

So to a good approximation we have

$$
\begin{aligned}
\varphi_{D} & =1+2 \pi i(\varphi-c) / \log (\varphi-c)+\ldots \\
\partial_{\varphi}{ }^{3} \varphi_{D} & =\frac{2 \pi i}{((\varphi-c) \log (\varphi-c))^{2}}+\ldots .
\end{aligned}
$$

Hence, when $\varphi$ is close to $c$, we have

$$
\begin{aligned}
& \sum_{n} 2 \pi i n^{4}(-1)^{n+1} d_{n} e^{2 \pi i n \varphi} \approx \\
& \approx \int d n 2 \pi i n^{4}(-1)^{n+1} d_{n} e^{2 \pi i n \varphi} \approx \frac{2 \pi i}{((\varphi-c) \log (\varphi-c))^{2}} .
\end{aligned}
$$

If we use the ansatz that $d_{n} \sim n^{a}(\log n)^{b} e^{-2 \pi i c n}$, then a simple saddle point calculation yields

$$
d_{n} \approx(2 \pi)^{3 / 2} n^{-3}(\log n)^{-2}(-1)^{n} e^{-2 \pi i c n+1} .
$$

To find $c$ note that we have to match the behaviour of $\varphi$ and $\varphi_{D}$ at the conifold point with their behaviour at the $E_{8}$ point $u=0$. The behaviour is matched by noting that [6]

$$
\begin{aligned}
\varphi_{D} & =\kappa \int \frac{d u}{u}\left(\xi F_{0}(u)+\frac{1}{\xi} F_{1}(u)\right) \\
\varphi & =\kappa \int \frac{d u}{u}\left(e^{2 \pi i / 3} \xi F_{0}(u)+\frac{e^{-2 \pi i / 3}}{\xi} F_{1}(u)\right),
\end{aligned}
$$

where $\kappa=i 3^{1 / 4} /\left(4 \pi^{3 / 2}\right)$ and $\xi=-i 3^{1 / 4} \Gamma(1 / 3)^{3} /\left(2^{2 / 3} \pi^{3 / 2}\right)$ and

$$
F_{0}(u)=u_{2}^{1 / 6} F_{1}\left(\frac{1}{6}, \frac{1}{6} ; \frac{1}{3} ; u\right) \quad F_{1}(u)=u_{2 / 6}^{5} F_{1}\left(\frac{5}{6}, \frac{5}{6} ; \frac{5}{3} ; u\right) .
$$


To find the integration constants, we know that as $u \rightarrow 0, \varphi \rightarrow 0$ and $\varphi_{D} \rightarrow 0$. Hence, the integrals in 5.13 are integrated from $u=0$ to $u$. Therefore, $c$ is given by

$$
\begin{aligned}
c & =\kappa \int_{0}^{1} \frac{d u}{u}\left(e^{2 \pi i / 3} \xi F_{0}(u)+\frac{e^{-2 \pi i / 3}}{\xi} F_{1}(u)\right) \\
& =-\frac{1}{2}+i\left(\left(\frac{9 \Gamma(1 / 3)^{3}}{2^{5 / 3} \pi^{3}}\right){ }_{3} F_{2}\left(\frac{1}{6}, \frac{1}{6}, \frac{1}{6} ; \frac{1}{3}, \frac{7}{6} ; 1\right)-\frac{\sqrt{3}}{2}\right),
\end{aligned}
$$

where ${ }_{3} F_{2}$ is a generalized hypergeometric function evaluated at $u=1$. Hence we find that

$$
d_{n} \approx(2 \pi)^{3 / 2} n^{-3}(\log n)^{-2} \exp ((2 \pi-\alpha) n-1),
$$

where $\alpha$ is found from 5.15 and is approximately $\alpha=.451967$. Comparing this with 5.6, we see that the naive result is not such a terrible approximation.

\section{Acknowledgements}

We would like to thank W. Lerche and P. Mayr for valuable discussions. N.W. is also grateful to the ITP in Santa Barbara, and the Institute for Advanced Study in Princeton for hospitality while this work was being done. This work is supported in part by funds provided by the DOE under grant number DE-FG03-84ER-40168, and by the National Science Foundation under grant No. PHY94-07194.

\section{References}

[1] O. Ganor and A. Hanany, Nucl. Phys. B474 (1996) 122; N. Seiberg and E. Witten, Nucl. Phys. B471 (1996) 121; M. Duff, H. Lu and C.N. Pope, Phys. Lett. B378 (1996) 101; M.R. Douglas, S. Katz and C. Vafa, Nucl. Phys. B497 (1997) 155; E. Witten, Mod. Phys. Let. A11 (1996) 2649.

[2] O. Ganor, Nucl. Phys. B479 (1996) 197.

[3] A. Klemm, P. Mayr and C. Vafa, "BPS States of Exceptional NonCritical Strings", CERN-TH-96-184, hep-th/9607139.

[4] D. R. Morrison and C. Vafa, Nucl. Phys. B473 (1996) 74; Nucl. Phys. B476 (1996) 437.

[5] E. Witten, Nucl. Phys. B471 (1996) 195.

[6] W. Lerche, P. Mayr and N. P. Warner, Nucl. Phys. B499 (1997) 125. 
[7] J. Minahan, D. Nemeschansky and N. P. Warner, "Investigating the BPS Spectrum of Non-Critical $E_{n}$ Strings", USC-97/006, NSF-ITP-97055, hep-th/9705237.

[8] M. Bershadsky, S. Cecotti, H. Ooguri and C. Vafa, Comm. Math. Phys. 165 (1994) 311.

[9] O. Ganor, D. Morrison and N. Seiberg, Nucl. Phys. B487 (1997) 93.

[10] A. Klemm, W. Lerche, P. Mayr, C. Vafa and N. P. Warner, Nucl. Phys. B477 (1996) 746.

[11] M. Green, J. Schwarz and E. Witten, "Superstring Theory: 1", Cambridge University Press 1987. 